\title{
The thickness of the ventral medial prefrontal cortex (vmPFC) predicts the self-prioritization effect (SPE) on the interaction between spatial reference frames and spatial domains
}

Jie Huang

Soochow University

Xiaoyu Tang

Liaoning Normal University

Aijun Wang ( $\sim$ ajwang@suda.edu.cn )

Soochow University

Ming Zhang

Soochow University

\section{Research Article}

Keywords: Self-prioritization effect, Reference frame, Near and far spaces, Structural MRI

Posted Date: October 27th, 2021

DOl: https://doi.org/10.21203/rs.3.rs-970699/v1

License: (c) (1) This work is licensed under a Creative Commons Attribution 4.0 International License.

Read Full License 


\section{Abstract}

Neuropsychological studies have demonstrated that the preferential processing of near-space and egocentric representation is associated with the self-prioritization effect (SPE). However, relatively little is known concerning whether the SPE is superior to the representation of egocentric frames or near-space processing in the interaction between spatial reference frames and spatial domains. The present study adopted the variant of the shape-label matching task (i.e., color-label) to establish an SPE, combined with a spatial reference frame judgment task, to examine how the SPE leads to preferential processing of nearspace or egocentric representations. Surface-based morphometry analysis was also adopted to extract the cortical thickness of the ventral medial prefrontal cortex (VmPFC) to examine whether it could predict differences in the SPE at the behavioral level. The results showed a significant SPE, manifested as the response of self-associated color being faster than that of stranger-associated color. Additionally, the SPE showed a preference for near-space processing, followed by egocentric representation. More importantly, the thickness of the vmPFC could predict the difference in the SPE on reference frames, particularly in the left frontal pole cortex and bilateral rostral anterior cingulate cortex. These findings indicated that the SPE showed a prior entry effect for information at the spatial level relative to the reference frame level, providing evidence to support the structural significance of the self-processing region. The present study also further clarified the priority in SPE processing and role of the SPE within the real spatial domain.

\section{Introduction}

The ability to represent surrounding spatial information is an essential requirement of living beings, and individuals must represent spatial orientation information at all times in the real three-dimensional world. Generally, different spatial representation strategies have been used to represent spatial orientation information as quickly and accurately ${ }^{1}$, as possible and it has been suggested that people represent spatial information using egocentric (i.e., subject-to-object relations to form body-centered representations) and/or allocentric (i.e., object-to-object relations to form world-centered representations) reference frames ${ }^{2,3}$. Evidence from behavioral and neuropsychological studies has supported that individuals preferentially adopt different reference frames to represent spatial information in depth locations ${ }^{4-7}$.

Previous studies have suggested that the space domain is divided into near (within the range of the arm) and far (beyond the range of the arm) spaces based on the hand-reaching distance ${ }^{5,8,9}$. According to the perception/action modal, the dorsal stream is implicated in near-space processing when individuals conduct an action and manipulation representation, and the ventral stream is implicated in far-space processing when individuals conduct a perception representation ${ }^{10-13}$. Likewise, evidence from clinical studies has shown that the dorsal stream is involved in egocentric representation, and the ventral stream is involved in allocentric representation ${ }^{14-16}$. Specifically, egocentric representation is encoded as sensorimotor representations through the dorsal stream, and allocentric representations are encoded as perceptual representations through the ventral stream ${ }^{4,17-19}$. Based on perception/action modal, 
anatomically specified dorsal and ventral visual streams differentially support near- and far-space processing or allocentric and egocentric reference frames, respectively.

Studies on the interaction between spatial domains and reference frames have found that individuals show processing precedence for egocentric presentation and near-space processing ${ }^{4,6,7}$. Chen et al. $(2012 b)^{4}$ showed a faster response in egocentric tasks regardless of near or far space, indicating that participants preferred egocentric representation. The functional magnetic resonance imaging results revealed that the parietal-occipital junction (POJ) shows enhanced neural activity in near-space processing, revealing a near-space preference. Wang et al. (2016a) ${ }^{7}$ further supported the view that the POJ acts as an interface between the dorsal and ventral streams in near and far space processing, showing higher activity to the target in near space than in far space. Therefore, previous studies have demonstrated a priority processing egocentric rather than allocentric reference frames and near rather than far space representations when reference frames interact with spatial domains.

Because objects that unexpectedly approach the observer have higher self-relevancy and farther unexpected objects involve less self-related thoughts, previous studies have indicated that attentional reorienting along 3D space involves self-related processing ${ }^{20-23}$. In other words, because of the strong self-relevancy in near space, individuals tend to show a self-prioritization effect (SPE) in near-space processing. The SPE is the phenomenon in which individuals focus on self-related information or objects and respond faster (or more accurately) relative to information associated with others ${ }^{24-26}$. Therefore, compared with far-space processing, the SPE shows priority in near-space processing $5,27,28$. Converging evidence has established the SPE using a shape-label matching task ${ }^{26,29-31}$. They found a faster response of self-association pairings than stranger-association pairings and higher activation of the ventral medial prefrontal cortex (VmPFC) in self-referential processing ${ }^{24,32-37}$. Additionally, the SPE is the primary reason that egocentric representation in spatial processing is preferred ${ }^{16}$. Some studies have demonstrated that participants tend to adopt an egocentric bias (self-reference bias) to represent internal representation, as reflected in the priority of the SPE for egocentric representation ${ }^{38-40}$. Therefore, the precondition of the SPE is the unconscious use of egocentric reference frame representation.

Considering the role of the SPE in near space processing and egocentric representation, the present study was specifically designed to address how the SPE controls the interaction between spatial domains and spatial reference frames. Specifically, participants have addressed the conflict between performing allocentric visuospatial judgments on near space and egocentric visuospatial judgments on far space. Addressing this issue would allow better understanding of the priority of the SPE and clarify the role of the SPE within the real 3D spatial domain. In the present study, all the participants were randomly assigned to either the self-association group or stranger-association group, and both groups were required to complete the spatial reference frame task in near and far spaces. The self-association group only needed to respond to the self-associated object, while the stranger-association group only needed to respond to the stranger-associated object. To further investigate whether the cortical thickness of selfprocessing regions could predict the SPE on spatial reference frames, we set the vmPFC as a region of 
interest (ROI) and extracted the cortical thickness of the vmPFC using surface-based morphometry analysis to correlate with the performance in the spatial reference frame task. We hypothesized that the SPE could affect the performance of egocentric representation and near-space processing in the selfassociation group and showed a preference for the process of the object in near space, followed by egocentric representation. Additionally, the cortical thickness of the vmPFC might be correlated with the performance of near-space processing and egocentric representation.

\section{Methods}

Participants. A total of 108 undergraduate and graduate students (42 male; age: $21.13 \pm 1.95$ years) participated in the experiment. The participants were randomly assigned to the self-association group and stranger-association group, with fifty-four participants in each group. All the participants were righthanded and had normal hearing, vision (or corrected vision), and color vision, with no history of neurological or psychiatric disorders. None of the participants had participated in any similar experiment. The present study was conducted according to the Declaration of Helsinki and was approved by the Ethics Committee of Soochow University. All the participants provided written informed consent. The sample size was calculated using the G-Power 3.1 toolbox ${ }^{41,42}$. According to a previous study ${ }^{43}$, a hybrid design should have a medium effect size $(f=0.25)$. With $a=0.05$ and power $=0.80$, the appropriate sample size was calculated to be at least 82 . Nine participants were excluded from the statistical analysis because of lower accuracy.

Apparatus and materials. The behavioral experiment was conducted on a laptop computer (Lenovo ThinkPad E480) with a screen resolution of $1024 \times 768$ pixels and a refresh rate of $60 \mathrm{~Hz}$. The experiment comprised a delayed match-to-sample task (i.e., color-label matching) and a spatial reference frame task (Figure 1a). The color-label matching task was a modified version of the shape-label association task used by Sui et al. (2012) ${ }^{44}$. During the color-label matching task, the visual stimuli were presented on a laptop screen with a gray background (RGB: 125, 125, 125) in E-prime 2.0 software. The visual stimuli comprised colored forks (e.g., black and white) and social labels (e.g., you and stranger). The black fork (RGB: $0,0,0)$ and white fork (RGB: $255,255,255)$ were presented above a fixation cross $\left(0.8^{\circ} \times 0.8^{\circ}\right)$ at the center of the screen (see Figure $1 \mathrm{~b})$. The word "you" or "stranger" $\left(3.1^{\circ} / 1.6^{\circ}\right)$ was displayed below the fixation cross. The distance between the center of the color or word and fixation cross was $3.5^{\circ}$.

The spatial reference frame judgment task was a modified version of the virtual spatial reference frame judgment task used by Chen et al. (2012b) ${ }^{4}$. The spatial reference frame judgment task involved two parts. The first part was a reference frame judgment task (i.e., egocentric and allocentric) in near space. The second part was a reference frame judgment task in the far space. During the spatial reference frame judgment task (Figure 1c), the stimuli in near space were presented on the laptop screen with a gray background (RGB: 125, 125, 125) in Presentation software (Neurobehavioral Systems Inc.), and the stimuli in the far space were presented on an EPSON CB-X29 projector under the same conditions. The visual stimuli comprised a colored fork (black or white) with a radius angle of $2.5^{\circ}$ intersecting an orange plate (RGB: $220,75,30$ ) with a radius angle of $15^{\circ}$. The fork could appear at one of four egocentric 
positions. For each of the four egocentric locations of the fork $\left(-5^{\circ},-3.5^{\circ}, 3.5^{\circ}\right.$, or $\left.5^{\circ}\right)$, the location of the plate varied independently around the fork, using four possible allocentric positions $\left(-2.4^{\circ},-1.7^{\circ}, 1.7^{\circ}\right.$, or $\left.2.4^{\circ}\right)$. The monitor (projector) was viewed at distances of 59 centimeters and 3.78 meters in the near- and far-space tasks, respectively. The visual angles of the egocentric and allocentric distances were both matched for near and far spaces.

Design and procedure. The color-label matching task was a 2 (association: self vs. stranger) $\times 2$ (matching: matched vs. not matched) within-subjects design, divided into a training stage and a matching stage. First, in the training stage, the participants were required to code colored forks (black or white) as self or stranger. Specifically, the participants were told, "you are represented by a black fork, and a stranger is represented by a white fork". After that, the participants had to judge whether the color-label pairings were correct in the matching stage. Specifically, each trial started with the presentation of a central fixation cross for $500 \mathrm{~ms}$. Next, a pairing of color and label (you or stranger) was presented for $100 \mathrm{~ms}$. The participants had to determine whether the color was correctly assigned to the person as accurately and quickly as possible within the timeframe (ranging from 800 to $1200 \mathrm{~ms}$ ). The feedback (e.g., correct, wrong, or too slow) was presented for $500 \mathrm{~ms}$ at the end of each trial. The participants were required to perform 240 trials over three blocks, and they were informed of their overall accuracy at the end of the block. All the participants were explicitly informed that they could only conduct the spatial reference frame judgment tasks when their overall accuracy was higher than $90 \%$. The color-label matching task served as training to make the participants master the color-label associations. All the pairing conditions were counterbalanced across participants.

The spatial reference frame judgment task was a 2 (spatial domains: near vs. far) $\times 2$ (reference frame judgment tasks: allocentric vs. egocentric) $\times 2$ (groups: self-association vs. stranger-association) hybrid design. This task had two stages. First, the participants were randomly assigned to either the selfassociation group or stranger-association group. The self-association group was required to judge the position of the self-associated fork, and the stranger-association group was required to judge the position of the stranger-associated fork. Both groups were provided with verbal instruction that was the same as that provided in the color-label matching task (e.g., you are the white fork, the stranger is represented by the black fork). In the egocentric judgment task, each trial started with the presentation of instructions for $3000 \mathrm{~ms}$. Afterward, the stimulus (a colored fork intersecting an orange plate) was presented for $150 \mathrm{~ms}$. The participants had to determine whether the self- or stranger-association fork was on the left or right side of the midline of their body by pressing the response button with the right index finger or middle finger as accurately and quickly as possible within $1500 \mathrm{~ms}$. The duration of each trial was 1650 ms with a $250 \mathrm{~ms}$ interstimulus interval. In the allocentric judgment task, the participants were required to determine whether the fork was on the left or right side of the midline of the plate. Except for the task instruction, the procedure of the allocentric representation task was the same as that of the egocentric judgment task. The participants needed to complete 384 trials of egocentric and allocentric judgment tasks in near and far spaces. Presentation order was counterbalanced across stimuli, tasks, spatial locations, and groups. In addition, the visual angle between the near space and far space was counterbalanced across participants. To ensure that the midline of participants was aligned with the 
midline of the monitor, the participants' head position was stabilized using a chin rest throughout the experiment.

MRI acquisition. The participants were scanned on a 3.0 T Magnetom Prisma scanner with a commercial 64-element sensitivity encoding head coil array. For each participant, T1-weighted volumes were acquired using a magnetization-prepared gradient echo (MPRAGE) in an MRI room (number of layers $=36$; slice thickness $=1 \mathrm{~mm}$; scanning time $=5 \mathrm{~min} ; \mathrm{FOV}=256 \times 256 \mathrm{~mm}$; scanning matrix size: $256 \times 256 \times 256$; $\mathrm{TR} / \mathrm{TE}=2300 / 2.34 \mathrm{~ms}$; flip angle $=8^{\circ}$ ).

T1-weighted image preprocessing and processing. The original T1-weighted images were converted from the DICOM format to the NIfTI format using MRIcroN software (dcm2niigui toolbox; https://www.nitrc.org/projects/mricron). After that, the oriented and neck-cut T1-weighted images were processed to obtain cortical thickness measures using FreeSurfer 6.0 software. FreeSurfer provides a full processing stream for T1-weighted MR images, including the removal of nonbrain tissue, automated Talairach transform computation, intensity normalization, skull stripping, white matter segmentation, filling and cutting, smoothing, inflation, spherical registration, and cortical parcellation statistics (https://surfer.nmr.mgh.harvard.edu/fswiki).

Brain structure measurements for cortical thickness were obtained using the semiautomated segmentation tool FreeSurfer. Cortical thickness maps were created using both signal intensity and continuity information from the 3D volume of magnetic resonance images, where the thickness was calculated as the closest distance from a pial to the white matter boundary at each vertex ${ }^{45,46}$. To avoid the misregistration of gray and white matter voxels, the quality of processed volumes was visually checked slice by slice using the FreeView toolbox before extracting the cortical thickness of the ROIs. Thickness maps were spatially smoothed using a Gaussian kernel with a half maximum width of $10 \mathrm{~mm}$. The maps were then averaged across the participants using a spherical aligning method for cortical folding patterns. All the operations to calculate cortical thickness were performed using code commands (https://surfer.nmr.mgh.harvard.edu/fswiki/FreeSurferCommands). Because the vmPFC acts as an ROI, the cortical thickness of the vmPFC in the medial orbitofrontal cortex (OFC), rostral and caudal anterior cingulate cortex (ACC), insular cortex, and frontal pole cortex (FPC) of the left and right hemispheres were extracted ${ }^{47,48}$.

\section{Results}

Behavioral data. To ensure that the samples were representative and unbiased, nine participants were excluded from the statistical analysis because they had low accuracy (lower than 90\%) in the spatial reference frame judgment task, possibly because of the lack of attention or motivation during the experiment. Because the overall accuracy of all the participants was high (all above 94\%), the present study focused more on reaction time (RT) in the statistical analysis. 
For the RT data, 2 (groups: self-association vs. stranger-association) $\times 2$ (spatial domains: near vs. far) $\times$ 2 (reference frame judgment tasks: allocentric vs. egocentric) mixed ANOVA was conducted (see Figure 2). The main effect of groups was significant, $F(1,97)=11.10, p=0.001, \eta_{p}^{2}=0.10$, and the RTs of the self-association group (592 ms) were faster than those of the stranger-association group (645 ms), indicating a significant SPE in the self-association group. The main effect of spatial domains was not significant, $F<1$. The main effect of reference frame judgment tasks was significant, $F(1,97)=35.02, p<$ $0.001, \eta_{p}^{2}=0.27$, and the RTs of the allocentric judgment task (604 ms) were significantly faster than those of the egocentric judgment task $(633 \mathrm{~ms})$. The interaction effect between groups and spatial domains was significant, $F(1,97)=61.70, p<0.001, \eta_{p}^{2}=0.39$. The interaction effect between groups and reference frame judgment tasks was significant, $F(1,97)=4.89, p=0.03, \eta_{p}^{2}=0.05$. The interaction effect between the spatial domains and reference frame judgment tasks was not significant, $F(1,97)=1.12, p=$ 0.29 . The three-way interaction effect among groups, spatial domains, and reference frame judgment tasks was significant, $F(1,97)=4.59, p=0.04, \eta_{p}^{2}=0.05$. To investigate the potential interaction between ownership groups and reference frames in near and far spaces, further simple effect analyses should be conducted.

First, to examine the role of groups in the three-way interaction effect, 2 (spatial domains: near vs. far) $\times 2$ (reference frame judgment tasks: allocentric vs. egocentric) repeated ANOVA was conducted in the selfassociation group and stranger-association group. For the self-association group, the main effect of spatial domains was significant, $F(1,49)=25.57, p<0.001, \eta_{p}{ }^{2}=0.33$, and the RTs of near-space processing $(579 \mathrm{~ms})$ were significantly faster than those of far-space processing (606 $\mathrm{ms})$. The main effect of reference frame judgment tasks was significant, $F(1,49)=6.35, p=0.02, \eta_{p}{ }^{2}=0.12$, and the RTs of the allocentric judgment task (583 ms) were significantly faster than those of the egocentric judgment task (602 ms). The interaction effect between spatial domains and reference frame judgment tasks was not significant, $F<1$. To examine whether the participants performed better in allocentric judgment tasks in near space or egocentric judgment tasks in far space, paired sample $t$-test was conducted in the selfassociation group. The RTs of the allocentric judgment task in near space (571 ms) were faster than those of the egocentric judgment task in far space $(617 \mathrm{~ms}), t(50)=5.02, p<0.001$, Cohen's $d=0.56$. For the stranger-association group, the main effect of spatial domains was significant, $F(1,48)=38.62, p<$ $0.001, \eta_{p}{ }^{2}=0.45$, and the RTs of near-space processing $(629 \mathrm{~ms})$ were faster than those of far-space processing $(660 \mathrm{~ms})$. The main effect of reference frame judgment tasks was significant, $F(1,48)=36.16$, $p<0.001, \eta_{p}{ }^{2}=0.43$, and the RTs of the allocentric judgment task $(624 \mathrm{~ms})$ were faster than those of the egocentric judgment task $(665 \mathrm{~ms})$. The interaction effect between spatial domains and reference frame judgment tasks was significant, $F(1,48)=4.24, p<0.045, \eta_{p}{ }^{2}=0.08$. Further analysis found that the RTs of the allocentric judgment task (636 ms) were faster than those of the egocentric judgment task (685 $\mathrm{ms}$ ) in far space, $F(1,48)=34.41, p<0.001, \eta_{p}{ }^{2}=0.42$, and the RTs of the allocentric judgment task (613 $\mathrm{ms}$ ) were also faster than those of the egocentric judgment task $(645 \mathrm{~ms})$ in near space, $F(1,48)=19.66$, $p<0.001, \eta_{p}{ }^{2}=0.29$, exhibiting faster allocentric processing. These findings indicated that individuals 
showed a preference for allocentric representation in near space compared with egocentric representation in far space.

Second, to examine the role of reference frame judgment tasks in the three-way interaction effect, a 2 (groups: self-association vs. stranger-association) $\times 2$ (spatial domains: near vs. far) mixed ANOVA was conducted in the egocentric and allocentric judgment tasks. For the egocentric judgment task, the main effect of groups was significant, $F(1,97)=12.57, p=0.001, \eta_{p}{ }^{2}=0.12$, and the RTs of the self-association group (602 ms) were significantly faster than those of the stranger-association group ( $665 \mathrm{~ms}$ ). The main effect of spatial domains was not significant, $F<1$. The interaction effect between groups and spatial domains was significant, $F(1,97)=41.37, p<0.001, \eta_{p}^{2}=0.30$. Further analysis found that the RTs of the self-association group in near space $(587 \mathrm{~ms})$ were faster than those of the stranger-association group (685 ms), $F(1,97)=26.68, p<0.001, \eta_{p}^{2}=0.22$, and no significant difference was found in the RTs between self-association (617 ms) and stranger-association (645 ms) groups in far space. For the allocentric judgment task, the main effect of groups was significant, $F(1,97)=7.61, p=0.007, \eta_{p}^{2}=0.07$, the RTs of the self-association group (583 ms) significantly faster than those of the stranger-association group (624 ms). The main effect of spatial domains was not significant, $F<1$. The interaction effect between groups and spatial domains was significant, $F(1,97)=49.42, p<0.001, \eta_{p}^{2}=0.34$. Further analysis found that the RTs of the self-association group in near space $(571 \mathrm{~ms})$ were faster than those of the stranger-association group (636 ms), $F(1,97)=18.06, p<0.001, \eta_{p}^{2}=0.16$, and no significant difference was found in the RTs between self-association ( $595 \mathrm{~ms}$ ) and stranger-association (613 ms) groups in far space. The above findings revealed that the SPE showed strongly affected near-space processing compared with far-space processing.

Third, to examine the role of spatial domains in the three-way interaction effect, a 2 (groups: selfassociation vs. stranger-association) $\times 2$ (reference frame judgment tasks: allocentric vs. egocentric) mixed ANOVA was conducted in near and far spaces. In the far space condition, the main effect of groups was not significant, $F(1,97)=2.02, p=0.16$. The main effect of reference frame judgment tasks was significant, $F(1,97)=27.12, p<0.001, \eta_{p}^{2}=0.22$, and the RTs of the allocentric judgment tasks (604 $\mathrm{ms}$ ) were significantly slower than those of the egocentric judgment tasks $(631 \mathrm{~ms})$. The interaction effect between groups and reference frame judgment tasks was not significant, $F(1,97)=1.17, p=0.28$. In the near space condition, the main effect of groups was significant, $F(1,97)=25.46, p<0.001, \eta_{p}{ }^{2}=$ 0.21 , and the RTs of the self-association group ( $579 \mathrm{~ms}$ ) were significantly faster than those of the stranger-association group (660 ms). The main effect of reference frame judgment tasks was significant, $F(1,97)=28.65, p<0.001, \eta_{p}^{2}=0.23$, and the RTs of the allocentric judgment task (603 ms) were significantly faster than those of the egocentric judgment task $(636 \mathrm{~ms})$. The interaction effect between groups and reference frame judgment tasks was significant, $F(1,97)=7.50, p=0.007, \eta_{p}^{2}=0.07$. Further simple effect analysis showed that the RTs of the egocentric judgment task (685 ms) were slower than those of the allocentric judgment task (636 ms) in the stranger-association group, $t(48)=5.87, p<0.001$, Cohen's $d=0.54$. However, no significant difference was found between allocentric ( $571 \mathrm{~ms})$ and egocentric (587 ms) judgment tasks in the self-association group, $t(49)=1.81, p=0.08$. To further 
examine whether the SPE was more beneficial for egocentric representation or allocentric representation, we obtained the reaction time difference ( $\mathrm{RT}$ allocentric-egocentric) by comparing the RT difference between allocentric and egocentric judgment tasks in near space. The independent-sample $t$-test results showed a significant difference between the self-association group and stranger-association group, $t(97)=2.74, p=$ 0.007 , Cohen's $d=0.55$, and the RT difference in the self-association group (16 ms) was less than that in the stranger-association group ( $49 \mathrm{~ms}$ ), indicating that the self-association group had a faster response on the egocentric judgment task in near space. These findings suggested that the SPE only benefited egocentric representation more than allocentric representation.

SMRI Correlation analysis. As discussed in the Introduction section, the vmPFC region is supported by self-referential material ${ }^{24}$. Additionally, the behavioral results benefited the SPE more on the allocentric reference frame in near space than on the egocentric reference frame in far space. Therefore, we focused on examining whether the cortical thickness of the vmPFC (i.e., OFC, ACC, FPC, and insular cortex) could predict the preference of the SPE on reference frames.

Pearson correlation analysis was conducted between the RTs of allocentric judgment tasks in near space and thickness of the vmPFC. Only the thickness of the left FPC was negatively associated with the RTs of the allocentric judgment task (see Figure 3a), $r(50)=-0.30, p=0.036$; other regions showed little or no significant associations with RTs of the allocentric judgment tasks ( $p s>0.05)$. Likewise, Pearson correlation analysis showed that, the thickness of the left rostral ACC was positively correlated with the RTs of egocentric judgment tasks in far space (see Figure $3 b), r(50)=0.32, p=0.026$; the thickness of the right rostral ACC was also positively correlated with the RTs of egocentric judgment tasks in far space (see Figure $3 c), r(50)=0.28, p=0.049$. A significant association was no longer found between the thickness and RTs of egocentric judgment task ( $p s>0.05)$.

\section{Discussion}

The present study used the color-label matching paradigm to establish an SPE and combined a conjunction visual search task to probe how the SPE influenced the judgment of the reference frames in different spatial domains. Participants were randomly divided into the self-association group and stranger-association group. The self-association group was required to respond to the object associated with themselves, while the stranger-association group was required to respond to the object associated with strangers. As expected, a significant SPE was found in the self-association group. Besides, the SPE showed preference processing for the allocentric reference frame in near-space compared with the egocentric reference frame in far space, revealing a preference for near-space processing, followed by egocentric representation. More importantly, correlation analysis showed a significant correlation between the cortical thickness of the vmPFC and RTs of reference frame tasks in near and far spaces, indicating that the thickness of the vmPFC could predict the difference in the SPE on reference frames.

First, the present study found a robust SPE in the self-association group compared with that in the stranger-association group. More specifically, the RTs of the spatial reference frame judgment task in the 
self-association group (592 ms) were significantly faster than those in the stranger-association group (645 ms). In accordance with the present study, previous studies have demonstrated that self-relevance automatically enhances stimulus processing, particularly in the form of a shape-label matching paradigm $44,49,53$. Importantly, the present study modified the associative-learning task and adopted the color-label matching task to make participants familiar and inform them about a stable color-label association, an approach that was different from the classic shape-label matching paradigm ${ }^{29-31}$. Yin and colleagues (2019) ${ }^{54}$ first adopted this novel color-label matching paradigm to establish self-/friend-/stranger-association in a spatial working memory task, and participants responded faster to the working memory probes of self-associated colors those of friend-associated and stranger-associated colors. Recently, their following study replicated the SPE in the matching paradigm ${ }^{55}$. Thus, these findings suggest that the SPE in the self-association matching paradigm works at a conceptual level, regardless of the attribution of associated stimuli ${ }^{56}$.

Second, consistent with the findings of previous studies of a close relationship among the SPE, nearspace processing, and egocentric reference frame ${ }^{4,38-40,57-60}$, the present study found that the SPE affected the interaction between spatial reference frames and spatial domains, as expected. More importantly, the SPE showed a preference for information of the allocentric reference frame in near space compared with the egocentric reference frame in far space. Specifically, the performance of the allocentric judgment task in near space was faster than that of the egocentric judgment task in far space in the self-association group, with performance exhibiting faster RTs (571 ms vs. $617 \mathrm{~ms}$ ). Moreover, further simple effect analysis suggested that the SPE was only observed in near-space processing, particularly in egocentric judgment tasks. Specifically, the performance of near-space processing (579 $\mathrm{ms}$ ) was faster than that of far-space processing $(606 \mathrm{~ms})$ in the self-association group, and improvement of the SPE on the egocentric reference frame was better than that on the allocentric reference frame (16 ms vs. $49 \mathrm{~ms}$ ) in near space. Therefore, we observed that the SPE benefited egocentric representation more based on near space, a finding that has not previously been described. In other words, near-space precedence was observed compared with egocentric representation during the information processing between spatial-level and reference frame-level information, revealing a near prior entry effect.

Here, we believe that at least two factors that should be considered for this consequence. On the one hand, unconscious exogenous attention to objects in near space may explain this consequence. Although the self-associated stimuli assigned increased personal significance and became perceptually more salient ${ }^{61}$, developing an egocentric-bias to represent objects in daily lives revealed that self-related objects (information) captured more endogenous attention than unfamiliar objects ${ }^{16,54,59}$. The participants focused more on the self-related stimuli cues when they adopted the egocentric strategy in the egocentric reference frame judgment task. However, the processing of information in near space still automatically captures more attention than egocentric representation information. Many current views have posited that objects in near space tend to attract extensive attention and be processed preferentially, and objects in near space acquire high attentional priority and automatically draw exogenous 
attention ${ }^{60,62}$. For example, Abrams et al. (2008) ${ }^{62}$ argued that individuals tend to process objects in near space or those close to them because objects in near space automatically captured their attention, revealing a near prior entry effect. Likewise, Cosman and Vecera $(2010)^{58}$ suggested that the SPE caused individuals to preferentially process objects in near space because of the processing order determined by attention. Additionally, from the perspective of evolutionary psychology, the memory and visuospatial attention of humans are selective ${ }^{63}$. Focusing on stimuli in near space is important for an organism to survive and thrive, and individuals generally tend to focus on information or objects closely related to adaptability ${ }^{63,64}$, likely because information in near space is more urgent and threatening to individuals.

On the other hand, the neural mechanism of the SPE may be the other possible underlying consideration. Evidence from neuropsychological studies has implied that the POJ, a neural interface for integrating and processing different kinds of information, was significantly activated when the egocentric reference frame interacted with far space and the allocentric reference frame interacted with near space $4,7,22,23$. Moreover, the POJ showed enhanced neural activity in near-space processing ${ }^{4,7}$. Furthermore, because of the urgency and immediacy of objects in near space, near-space processing showed high self-relevancy. Chen et al. (2012a) found that the default-mode network, including the posterior cingulate cortex, orbital prefrontal cortex, and left angular gyrus, showed higher activation of attentional orientation when participants completed the task in near space ${ }^{57}$. Given that the region of the POJ overlapped with selfprocessing regions, a common neural mechanism likely exists between the SPE and near-space processing. Thus, the SPE has a prior entry effect in near space processing is not surprising.

Third, regarding correlation analysis, our results supported the view that the SPE was associated with the cortical midline structure, particularly the vmPFC $24,26,35,37$. Specifically, the thickness of the left FPC was negatively correlated with the RTs of the allocentric judgment task in near space, and the thickness of the bilateral rostral ACC was positively correlated with the RTs of the egocentric judgment task in far space, a finding that contrasted the closed relationship between the SPE and ACC. Cortical thickness measures whether the brain structure is damaged or reveals normal development in morphological analysis of brain structure ${ }^{65-68}$. Better development of the brain area is generally associated with a higher cortical thickness. For example, Fleming et al. (2016) indicated that the thicker was the right anterior cingulate cortex, the better was cognitive control performance ${ }^{66}$. Because of the dominance of spatial-level information processing, it is not surprising that the thicker is the PFC, the faster is the response of the allocentric reference frame in near space. However, notably, the positive correlation in the present study may be due to processing conflict between the information of the spatial level and reference frame level. Many studies have demonstrated that vmPFC activity (such as the ACC) is involved in various selfprocessing tasks ${ }^{30,32,34-37}$. The present study found superiority in information processing at the spatial level compared with the reference frame level. In other words, our brains prioritized processing far space information and then processed the information of the egocentric reference frame when the egocentric judgment task was conducted in far space. Therefore, an individual with a thicker thickness of ACC will have stronger competition (i.e., take more cost) in the information processing between far-space processing and egocentric representation, exhibiting slower RTs with thicker thickness. 
In conclusion, the present study revealed that the SPE could modulate the interaction between spatial domains and spatial reference frame representation. Additionally, the information at the spatial level before the reference frame level manifested with near-space processing first, followed by egocentric representation. Furthermore, the cortical thickness of the left FPC and bilateral ACC could predict the difference in the SPE on the reference frame. These findings reveal the role of the SPE in reference frames in the real three-dimensional world and provide evidence to support the relationship between the vmPFC and the SPE in humans regarding brain structure.

\section{Declarations}

\section{Author contributions}

AW and MZ designed the research. JH and XT performed the research. JH and XT

analyzed the data. $\mathrm{JH}$ and $\mathrm{AW}$ wrote the manuscript text. AW and $\mathrm{MZ}$ reviewed the manuscript.

\section{Declaration of Competing Interest}

The authors declare that they have no known competing for financial interests or personal relationships that could have appeared to influence the work reported in this paper.

\section{Acknowledgments}

This research was supported by the National Natural Science Foundation of China (31700939 and 31871092). AW was also supported by the MOE Project of Humanities and Social Sciences (17YJC190024) and Philosophy and Social Science Foundation of universities in Jiangsu Province (2017SJB1344).

\section{References}

1. Chan, E., Baumann, O., Bellgrove, M. A. \& Mattingley, J. B. Extrinsic reference frames modify the neural substrates of object-location representations., 51 (5), 781-788 (2013).

2. Arzy, S. \& Schacter, D. L. Self-agency and self-ownership in cognitive mapping. Trends in Cognitive Sciences, 23 (6), 476-487 (2019).

3. Colombo, D. et al. Egocentric and allocentric spatial reference frames in aging: A systematic review. Neuroscience \& Biobehavioral Reviews, 80, 605-621 (2017).

4. Chen, Q., Weidner, R., Weiss, P. H., Marshall, J. C. \& Fink, G. R. Neural interaction between spatial domain and spatial reference frame in parietal-occipital junction. Journal of Cognitive Neuroscience, 24 (11), 2223-2236 (2012b).

5. Lane, A. R., Ball, K., Smith, D. T., Schenk, T. \& Ellison, A. Near and far space: Understanding the neural mechanisms of spatial attention. Hum. Brain. Mapp, 34 (2), 356-366 (2013). 
6. Lane, A. R., Ball, K. \& Ellison, A. Dissociating the neural mechanisms of distance and spatial reference frames., 74, 42-49 (2015).

7. Wang, A., Li, Y., Zhang, M. \& Chen, Q. The role of parieto-occipital junction in the interaction between dorsal and ventral streams in disparity-defined near and far space processing. PLoS ONE, 11 (3), (2016a). e0151838

8. Berti, A. \& Frassinetti, F. When far becomes near: Remapping of space by tool use. Journal of Cognitive Neuroscience, 12 (3), 415-420 (2000).

9. Zanini, A. et al. (2021). Peripersonal and reaching space differ: Evidence from their spatial extent and multisensory facilitation pattern.Psychonomic Bulletin \& Review,1-12.

10. Goodale, M. A. \& Haffenden, A. Frames of reference for perception and action in the human visual system. Neuroscience \& Biobehavioral Reviews, 22 (2), 161-172 (1998).

11. Goodale, M. A. \& Milner, A. D. Separate visual pathways for perception and action. Trends in Neurosciences, 15 (1), 20-25 (1992).

12. Milner, A. D. \& Goodale, M. A. (2008). Two visual systems re-viewed. Neuropsychologia, 46(3),774785.

13. Milner, A. D. \& Goodale, M. A. The visual brain in action (OUP Oxford, 2006).

14. Andersen, R. A. \& Buneo, C. A. Intentional maps in posterior parietal cortex. Annual Review of Neuroscience, 25 (1), 189-220 (2002).

15. Bruno, N. When does action resist visual illusions? Trends in Cognitive Sciences, 5 (9), 379-382 (2001).

16. Jiang, Y. V. \& Swallow, K. M. Spatial reference frame of incidentally learned attention., 126 (3), $378-$ 390 (2013).

17. Ruotolo, F. et al. Neural correlates of egocentric and allocentric frames of reference combined with metric and non-metric spatial relations., 409, 235-252 (2019).

18. Ruggiero, G., Ruotolo, F., Orti, R., Rauso, B. \& lachini, T. Egocentric metric representations in peripersonal space: A bridge between motor resources and spatial memory. British Journal of Psychology, 112 (2), 433-454 (2021).

19. Wang, A. et al. Interaction between spatial domain and spatial reference frame in deaf and hearing populations. Acta Psychologica Sinica, 48 (2), 153 (2016b).

20. Gusnard, D. A. \& Raichle, M. E. Searching for a baseline: Functional imaging and the resting human brain. Nature Reviews Neuroscience, 2 (10), 685-694 (2001).

21. Fox, M. D. et al. (2005). The human brain is intrinsically organized into dynamic, anticorrelated functional networks. Proceedings of the National Academy of Sciences, 102(27), 9673-9678.

22. Corbetta, M., Patel, G. \& Shulman, G. L. The reorienting system of the human brain: From environment to theory of mind., 58 (3), 306-324 (2008).

23. Qin, P. \& Northoff, G. How is our self related to midline regions and the default-mode network? Neurolmage, 57 (3), 1221-1233 (2011). 
24. Schäfer, S. \& Frings, C. Searching for the inner self: evidence against a direct dependence of the selfprioritization effect on the ventro-medial prefrontal cortex. Experimental brain research, 237 (1), 247256 (2019).

25. Schäfer, S., Wentura, D. \& Frings, C. Distinctiveness effects in self-prioritization. Visual Cognition, 25 (1), 399-411 (2017).

26. Sui, J., Rotshtein, P. \& Humphreys, G. W. (2013). Coupling social attention to the self forms a network for personal significance. Proceedings of the National Academy of Sciences, 110(19), 7607-7612.

27. McPhee, A. M., Constable, M. D., Saccone, E. J. \& Welsh, T. N. (2021). The influence of location, ownership, and the presence of a coactor on the processing of objects.Canadian Journal of Experimental Psychology/Revue Canadienne de Psychologie Expérimentale.

28. Suh, J. \& Abrams, R. A. Reduced object-based perception in the near-hand space. Exp. Brain Res, 233 (12), 3403-3412 (2015).

29. Humphreys, G. W. \& Sui, J. The salient self: Social saliency effects based on self-bias. Journal of Cognitive Psychology, 27 (2), 129-140 (2015).

30. Humphreys, G. W. \& Sui, J. Attentional control and the self: the Self-Attention Network (SAN). Cognitive Neuroscience, 7 (1), 5-17 (2016).

31. Schäfer, S., Frings, C. \& Wentura, D. About the composition of self-relevance: Conjunctions not features are bound to the self. Psychon. Bull. Rev, 23 (3), 887-892 (2016).

32. Denny, B. T., Kober, H., Wager, T. D. \& Ochsner, K. N. A meta-analysis of functional neuroimaging studies of self-and other judgments reveals a spatial gradient for mentalizing in medial prefrontal cortex. Journal of Cognitive Neuroscience, 24 (8), 1742-1752 (2012).

33. Hu, C. et al. Distinct and common aspects of physical and psychological self-representation in the brain: A meta-analysis of self-bias in facial and self-referential judgements. Neuroscience \& Biobehavioral Reviews, 61, 197-207 (2016).

34. Kurczek, J. et al. Differential contributions of hippocampus and medial prefrontal cortex to selfprojection and self-referential processing., 73, 116-126 (2015).

35. Lieberman, M. D., Straccia, M. A., Meyer, M. L., Du, M. \& Tan, K. M. Social, self, (situational), and affective processes in medial prefrontal cortex (MPFC): Causal, multivariate, and reverse inference evidence. Neuroscience \& Biobehavioral Reviews, 99, 311-328 (2019).

36. Philippi, C. L., Duff, M. C., Denburg, N. L., Tranel, D. \& Rudrauf, D. Medial PFC damage abolishes the self-reference effect. Journal of Cognitive Neuroscience, 24 (2), 475-481 (2012).

37. Qin, P., Wang, M. \& Northoff, G. Linking bodily, environmental and mental states in the self-A threelevel model based on a meta-analysis. Neuroscience and Biobehavioral Reviews, 115, 77-95 (2020).

38. Cunningham, S. J., Brebner, J. L., Quinn, F. \& Turk, D. J. The self-reference effect on memory in early childhood. Child Dev, 85 (2), 808-823 (2014).

39. Cunningham, S. J. \& Turk, D. J. A review of self-processing biases in cognition. Quarterly Journal of Experimental Psychology, 70 (6), 987-995 (2017). 
40. Meyer, M. L. \& Lieberman, M. D. Why people are always thinking about themselves: Medial prefrontal cortex activity during rest primes self-referential processing. Journal of Cognitive Neuroscience, 30 (5), 714-721 (2018).

41. Faul, F., Erdfelder, E., Buchner, A. \& Lang, A. G. Statistical power analyses using G* Power 3.1: Tests for correlation and regression analyses. Behav. Res. Methods, 41 (4), 1149-1160 (2009).

42. Faul, F., Erdfelder, E., Lang, A. G. \& Buchner, A. G* Power 3: A flexible statistical power analysis program for the social, behavioral, and biomedical sciences. Behav. Res. Methods, 39 (2), 175-191 (2007).

43. Cohen, J. Statistical power analysis for the behavioral sciences (Academic press, 2013).

44. Sui, J., He, X. \& Humphreys, G. W. Perceptual effects of social salience: Evidence from selfprioritization effects on perceptual matching. Journal of Experimental Psychology: Human Perception and Performance, 38 (5), 1105 (2012).

45. Dale, A. M., Fischl, B. \& Sereno, M. I. Cortical surface-based analysis: I. Segmentation and surface reconstruction. Neurolmage, 9 (2), 179-194 (1999).

46. Gautam, P., Anstey, K. J., Wen, W., Sachdev, P. S. \& Cherbuin, N. Cortical gyrification and its relationships with cortical volume, cortical thickness, and cognitive performance in healthy mid-life adults. Behav. Brain. Res, 287, 331-339 (2015).

47. Striedter, G. F. Principles of brain evolution (Sinauer associates, 2005).

48. Murray, E. A., Wise, S. P. \& Graham, K. S. The Evolution of Memory Systems: Ancestors, anatomy, and adaptations (Oxford University Press, 2017).

49. Caughey, S. et al. (2020). Self-prioritization during stimulus processing is not obligatory. Psychological Research,1-6.

50. Frings, C. \& Wentura, D. Self-prioritization processes in action and perception. Journal of Experimental Psychology: Human Perception and Performance, 40 (5), 1737 (2014).

51. Sui, J., Sun, Y., Peng, K. \& Humphreys, G. W. The automatic and the expected self: Separating self-and familiarity biases effects by manipulating stimulus probability. Atten. Percept. Psychophys, 76 (4), 1176-1184 (2014).

52. Sui, J. \& Humphreys, G. W. Aging enhances cognitive biases to friends but not the self. Psychon. Bull. Rev, 24 (6), 2021-2030 (2017).

53. Sun, Y., Fuentes, L. J., Humphreys, G. W. \& Sui, J. (2016). Try to see it my way: Embodied perspective enhances self and friend-biases in perceptual matching. Cognition, 153,108-117.

54. Yin, S., Sui, J., Chiu, Y. C., Chen, A. \& Egner, T. Automatic prioritization of self-referential stimuli in working memory. Psychol. Sci, 30 (3), 415-423 (2019).

55. Yin, S., Bi, T., Chen, A. \& Egner, T. Ventromedial prefrontal cortex drives the prioritization of selfassociated stimuli in working memory. Journal of Neuroscience, 41 (9), 2012-2023 (2021).

56. Schäfer, S., Wentura, D. \& Frings, C. Self-prioritization beyond perception. Exp. Psychol, 62 (6), 415425 (2015). 
57. Chen, Q., Weidner, R., Vossel, S., Weiss, P. H. \& Fink, G. R. Neural mechanisms of attentional reorienting in three-dimensional space. Journal of Neuroscience, 32 (39), 13352-13362 (2012a).

58. Cosman, J. D. \& Vecera, S. P. Attention affects visual perceptual processing near the hand. Psychol. Sci, 21 (9), 1254-1258 (2010).

59. Klein, S. B. A role for self-referential processing in tasks requiring participants to imagine survival on the savannah. Journal of Experimental Psychology: Learning, Memory, and Cognition, 38 (5), 1234 (2012).

60. Spence, C. \& Parise, C. Prior-entry: A review. Consciousness and Cognition, 19 (1), 364-379 (2010).

61. Payne, B., Lavan, N., Knight, S. \& McGettigan, C. Perceptual prioritization of self-associated voices. British Journal of Psychology, 112 (3), 585-610 (2021).

62. Abrams, R. A., Davoli, C. C., Du, F., Knapp, I. I. I. \& Paull, D. W. H., \& (2008). Altered vision near the hands. Cognition, 107(3),1035-1047.

63. Buss, D. Evolutionary psychology: The new science of the mind (Psychology Press, 2015).

64. Klein, S. B., Cosmides, L., Tooby, J. \& Chance, S. Decisions and the evolution of memory: Multiple systems, multiple functions. Psychol. Rev, 109 (2), 306 (2002).

65. Erpelding, N., Moayedi, M. \& Davis, K. D. Cortical thickness correlates of pain and temperature sensitivity., 153 (8), 1602-1609 (2012).

66. Fleming, K. A., Heintzelman, S. J. \& Bartholow, B. D. Specifying associations between conscientiousness and executive functioning: Mental set shifting, not prepotent response inhibition or working memory updating. Journal of Personality, 84 (3), 348-360 (2016).

67. Kühn, S., Schubert, F. \& Gallinat, J. Structural correlates of trait anxiety: Reduced thickness in medial orbitofrontal cortex accompanied by volume increase in nucleus accumbens. Journal of Affective Disorders, 134 (1), 315-319 (2011).

68. Liem, F. et al. Reliability and statistical power analysis of cortical and subcortical FreeSurfer metrics in a large sample of healthy elderly. Neurolmage, 108, 95-109 (2015).

\section{Figures}


(a)

\begin{tabular}{|l|l|l|l|}
\hline Assocition learning stage & Assocition matching & Spatial judgment task training & sMRI scanning \\
\hline
\end{tabular}

Time

(b)

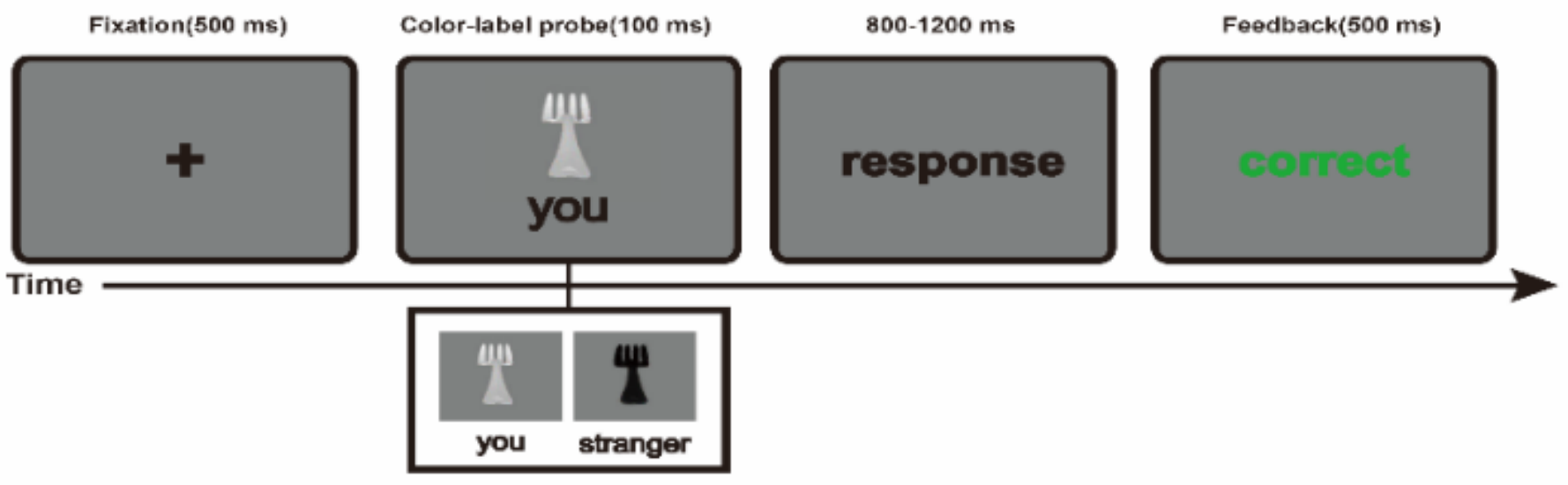

(c)

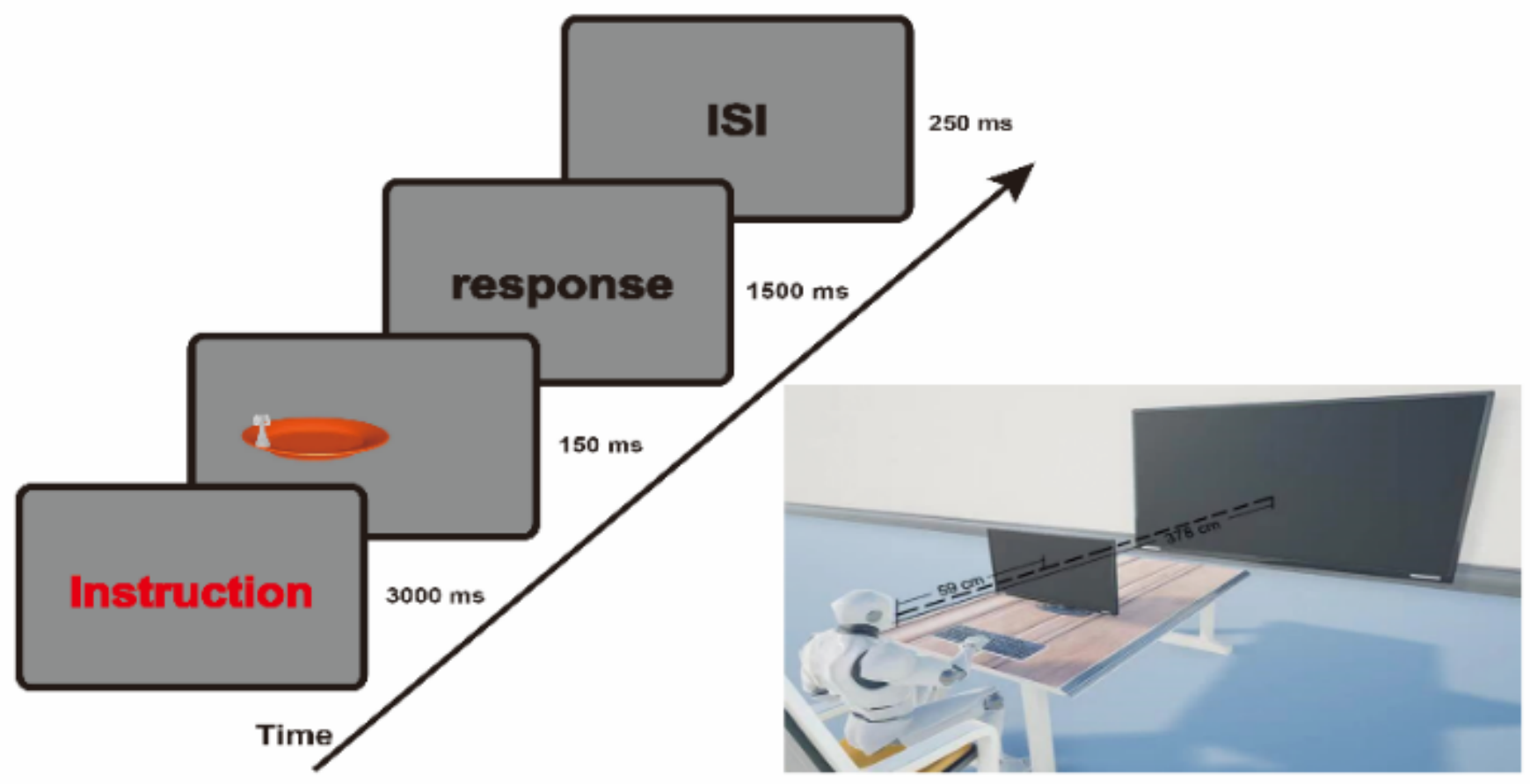

Figure 1

(a) Flowchart of the whole experiment. The overall experimental procedure comprised an association learning stage, an association training stage, the spatial reference frame judgment task, and structural MRI scanning. (b) Example stimuli and time of the single trial of the color-label matching task. The association between the color (black or white) and the label (you or stranger) was bound in the association learning stage. During the training stage, each trial started with a $500 \mathrm{~ms}$ duration fixation, followed by a probe stimulus for $100 \mathrm{~ms}$. The participants had to determine whether the color-label pairing matched as soon as possible within the timeframe ranging from 800 to $1200 \mathrm{~ms}$. (c) Procedure and temporal profile of stimulus presentation in the spatial reference judgment task. This task was 
presented block-by-block. Each block started with text guidance to inform participants of the type of task in the current block. The participants had to judge whether the fork was on the left or right side of the midline of their body in the egocentric judgment task and judge whether the fork was on the left or right side of the midline of the plate in the allocentric judgment task. Each block started with the presentation of instructions for $3000 \mathrm{~ms}$. The probe stimulus (a colored fork intersecting an orange plate) was presented for $150 \mathrm{~ms}$ afterward. The participants were asked to judge the position of the fork according to the instructions as accurately and quickly as possible within $1500 \mathrm{~ms}$. The duration of each trial was 1650 ms with a 250 ms interstimulus interval.

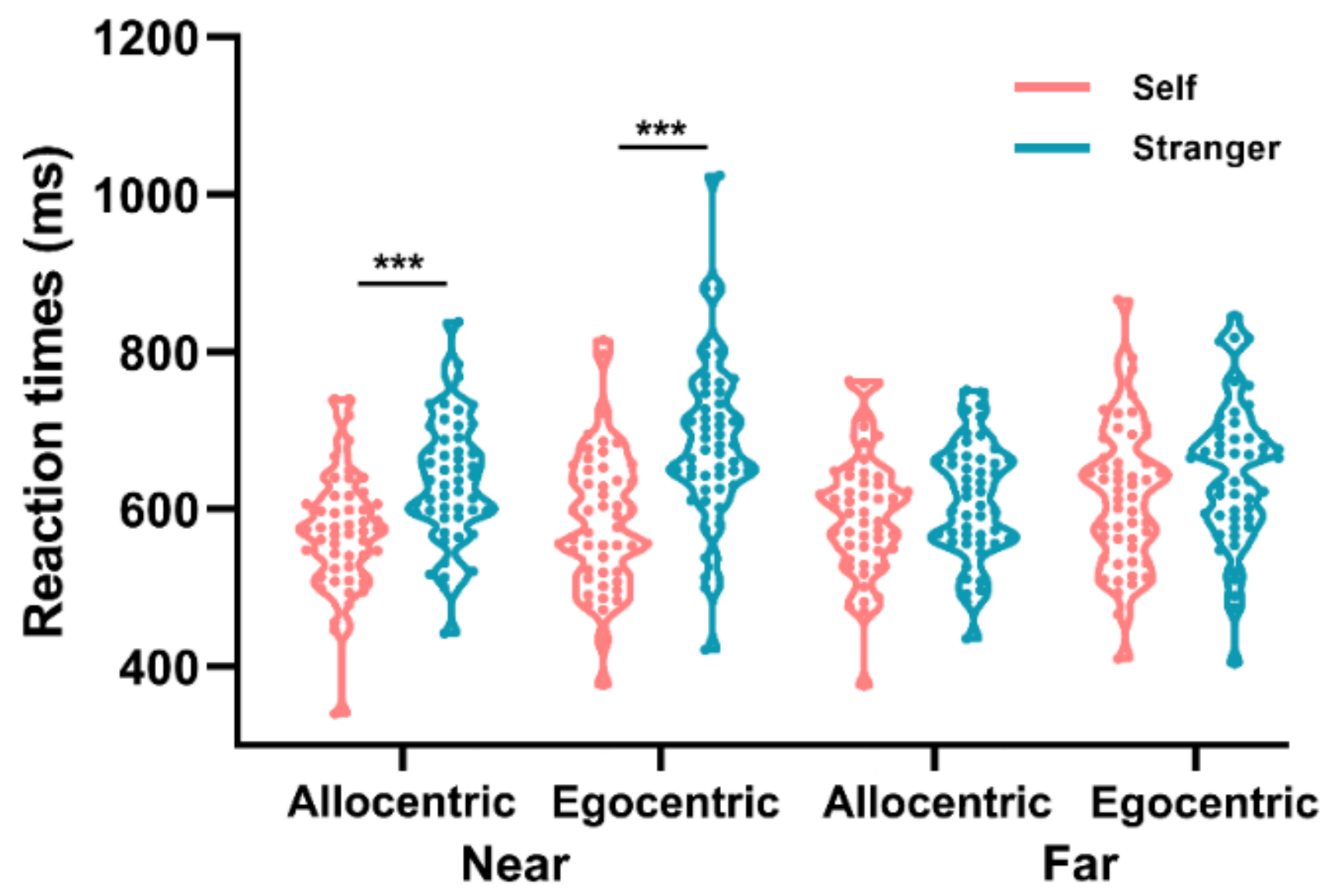

Figure 2

Mean reaction times in spatial reference frame tasks. The RT of the self-association group was faster than that of the stranger-association group $(p=0.001)$. Additionally, the RT of the allocentric judgment task in near space (571 ms) was faster than that of the egocentric judgment task in far space $(617 \mathrm{~ms})$ in the self-association group $(p<0.001)$. The RT of the allocentric judgment task was faster than that of the egocentric judgment task $(p<0.001)$. A significant interaction effect was found among groups, spatial domains, and reference frame judgment tasks. Specifically, in near space, the reaction times of allocentric representation in the self-association group $(571 \mathrm{~ms})$ were faster than those in the stranger-association group $(636 \mathrm{~ms})(p<0.001)$, and the reaction times of egocentric judgment tasks in the self-association group (587 ms) were faster than those in the stranger-association group $(685 \mathrm{~ms})(p<0.001)$. No 
difference was found in egocentric or allocentric judgment tasks between the groups in far space (ps > 0.05).

(a)

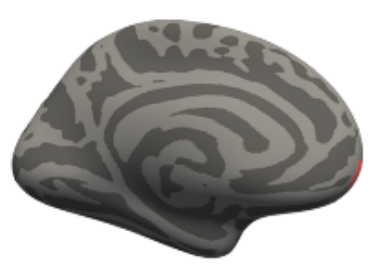

left PFC

(b)

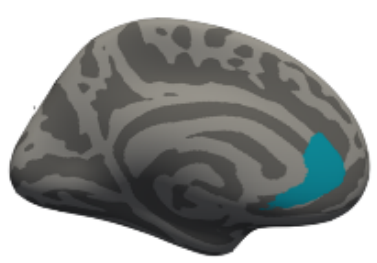

left rostral ACC

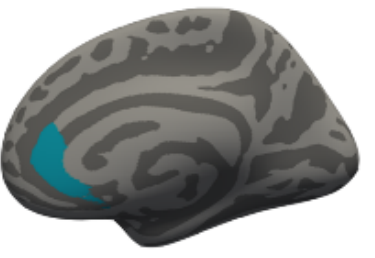

right rostral ACC

(c)
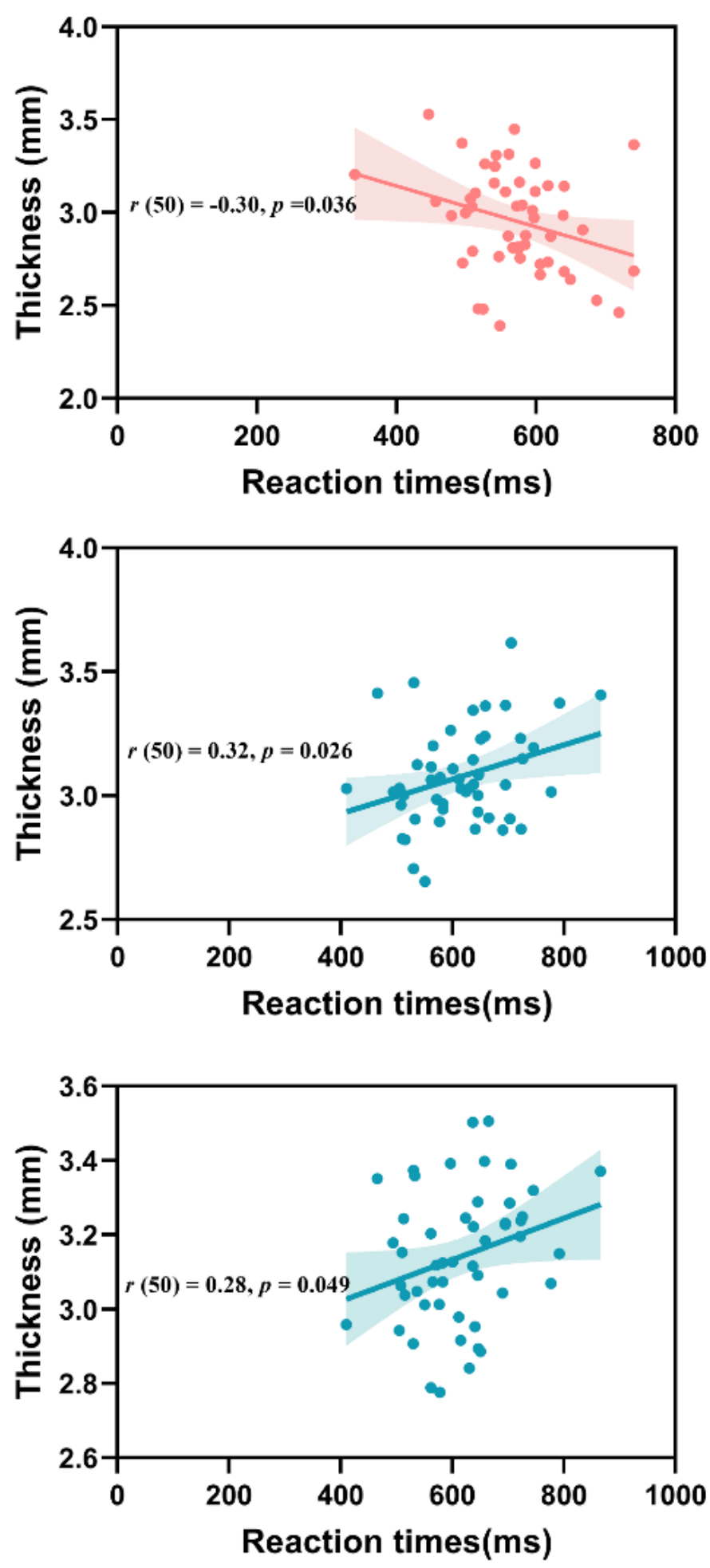

Figure 3

Behavioral SPE-related cortical thickness of ROIs. (a) The RTs of the allocentric judgment task in near space were negatively related to the thickness of the left frontal pole cortex $(p=0.036)$. (b) The RTs of the egocentric judgment task in far space were positively related to the thickness of the left rostral anterior 
cingulate cortex $(p=0.026)$. (c) The RTs of the egocentric judgment task in far space were positively related to the thickness of the right rostral anterior cingulate cortex $(p=0.049)$. 